\title{
Characterization of primary human skeletal muscle cells from multiple commercial sources
}

\author{
Jane Owens • Karen Moreira • Gerard Bain
}

Received: 2 March 2013 / Accepted: 24 June 2013 / Published online: 17 July 2013 / Editor: T. Okamoto

(C) The Author(s) 2013. This article is published with open access at Springerlink.com

\begin{abstract}
There is a significant unmet need for safe, anabolic muscle therapies to treat diseases and conditions associated with severe muscle weakness and frailty. The identification of such therapies requires appropriate cell-based screening assays to select compounds for further development using animal models. Primary human skeletal muscle cells have recently become available from a number of commercial vendors. Such cells may be valuable for studying the mechanisms that direct muscle differentiation, and for identifying and characterizing novel therapeutic approaches for the treatment of age- and injury-induced muscle disorders. However, only limited characterization of these cells has been reported to date. Therefore, we have examined four primary human muscle cell preparations from three different vendors for their capacity to differentiate into multinucleated myotubes. Two of the preparations demonstrated robust myotube formation and expressed characteristic markers of muscle differentiation. Furthermore, these myotubes could be induced to undergo morphological atrophy- and hypertrophy-like responses, and atrophy could be blocked with an inhibitor of myostatin signaling, a pathway that is known to negatively regulate muscle mass. Finally, the myotubes were efficiently infected with recombinant adenovirus, providing a tool for genetic modification. Taken together, our results indicate that primary human muscle cells can be a useful system for studying muscle differentiation, and may also provide tools for studying new therapeutic molecules for the treatment of muscle disease.
\end{abstract}

J. Owens $(\bowtie) \cdot \mathrm{K}$. Moreira $\cdot$ G. Bain

Tissue Repair Research Unit, Pfizer, 200 Cambridge Park Drive, Cambridge, MA 02140, USA

e-mail: Jane.Owens@Pfizer.com

Present Address:

G. Bain

Vertex Pharmaceuticals, 130 Waverly Street, Cambridge, MA

02139, USA
Keywords Skeletal muscle cell differentiation · Myoblast . Myotube $\cdot$ Hypertrophy $\cdot$ Atrophy

\section{Introduction}

Age- or injury-induced muscle weakness leading to frailty is a major public health problem that is predicted to escalate in the future as the number and proportion of older adults increase in the general population (Berger and Doherty 2010). There is an unmet need for therapeutic strategies that can slow the effects of aging on muscle function in the frail elderly so as to maintain or improve their quality of life. Identifying suitable therapeutic targets and testing candidate drugs for the ability to improve muscle function require cellbased model systems that reliably predict in vivo effects in both pre-clinical rodent models and human patients. A number of useful rodent cell lines such as mouse $\mathrm{C} 2 \mathrm{C} 12$ or rat L6 myoblasts are available (Sultan et al. 2006). These cell lines, and others, have been used extensively to explore the molecular mechanisms of muscle differentiation and function (McFarlane et al. 2006; Cui et al. 2009). In addition, they have been used in some drug discovery screens (Baudy et al. 2009; Cho et al. 2009). However, because immortalized cell lines often are genetically abnormal, and have been maintained under artificial conditions in culture for very long periods of time which can cause them to deviate from normal function, a potentially more predictive screening strategy would use primary human muscle cells. Such cells have recently become available from a number of commercial vendors, but only limited characterization of these various primary cells has been reported (Janowski 2011). Therefore, we examined several primary human skeletal muscle cell preparations from different vendors and characterized each for their capacity to reproducibly differentiate into multinucleated myotubes. Those that reproducibly differentiated into myotubes were further examined for the expression of 
various markers of skeletal muscle cells such as Myogenic Differentiation-1 (MYOD), Myocyte enhancer factor 2C (Mef2C), Myogenin (MYOG), Troponin T type 1 (TNNT), and Myosin heavy chain-2 (MYH2). We also tested known inducers of skeletal muscle atrophy (myostatin and dexamethasone) and hypertrophy (insulin-like growth factor-1) for effects on the differentiated myotubes derived from the primary cells. In addition, we found that myostatin-induced activity on the myotubes can be blocked by treatment with a soluble myostatin receptor, similar to one that is currently in clinical trials for diseases associated with muscle wasting (Lee et al. 2005; Pistilli et al. 2011). Finally, we show that both the undifferentiated primary muscle cells as well as the myotubes they give rise to can be infected with adenovirus. This observation suggests the potential for using these cells in genetic screens (both overexpression and knock-down) to identify factors that play a role in muscle differentiation and function. Such factors may provide novel targets for future drug development.

\section{Materials and Methods}

Cells, media, and supplement HSMM (catalog \#CC-2580, Lot\# 6F4528, sourced from quadriceps muscle of a 16-yr-old male cadaver) and SkMC-L (catalog \#CC-2561, Lot\# 6F3791, sourced from human fetal skeletal muscle) were purchased from Lonza (Walkersville, MD). SkMDC (catalog \#SK-1111, Lot\# P101014-50M2, sourced from rectus abdominus muscle of a 50-yr-old male Caucasian with a Body Mass Index (BMI) of 21, non-diabetic, smoker for $20 \mathrm{yr}$ ) came from Cook Myosite (Pittsburgh, PA). SkMC-P (catalog \#C12530, Lot\# 8121902.17, sourced from unspecified skeletal muscle of a 21-yr-old male Caucasian) was obtained from PromoCell (Heidelberg, Germany). Vendors also supplied growth media with supplements and serum for their respective cells. HSMM differentiation medium was prepared by adding $2 \%$ horse serum to DMEM-F12 medium (both from Invitrogen, Grand Island, NY). The vendor stated that HSMM would differentiate in all horse serum lots, so no pre-screening was required. SkMDC differentiation medium (ready to use) was supplied by the vendor (Catalog \# MD-5555). Recombinant human Myostatin (Mstn) and ActRIIb-Fc were generated in-house as previously described (Thies et al. 2001; Lee et al. 2005). Recombinant human IGF-1 and TWEAK were purchased from R\&D Systems (Minneapolis, MN), while Dex was obtained from Sigma (St. Louis, MO).

Cell culture and differentiation HSMM and SkMDC were maintained in growth medium with supplements and fetal bovine calf (FBS) serum provided by the vendors. Cultures were expanded and frozen stocks were prepared following the vendors' instructions. SkMDC were passaged when they reached 50\% confluence, (about every 2-3 d). When passaging, the culture medium was warmed to $37^{\circ} \mathrm{C}$ and the cells were seeded at $5,000-7,500$ cells $/ \mathrm{cm}^{2}$ and incubated in a humidified incubator at $37^{\circ} \mathrm{C}, 5 \% \mathrm{CO}_{2}$. Culture medium was changed every other day. HSMM were passaged when they reached $50-70 \%$ confluency (about every $3 \mathrm{~d}$ ). The culture conditions for these cells were the same as those for SkMDC except they were seeded at $3,500 \mathrm{cells} / \mathrm{cm}^{2}$. For both cell types, cultures were used for experiments after no more than seven population doublings because the cells' rate of differentiation declined after higher population doublings. To induce differentiation, cells were plated at 20,000cells $/ \mathrm{cm}^{2}$ in 12-well polystyrene cell culture plates (VWR) and incubated overnight in growth medium in a cell culture incubator $\left(37^{\circ} \mathrm{C}, 5 \% \mathrm{CO}_{2}\right)$. The following morning, the growth medium was replaced with differentiation medium and the cultures were incubated for $3 \mathrm{~d}$, during which time myotube differentiation occurred. To examine effects on differentiation, agents to be tested were added at the time of medium change and cultured for $3 \mathrm{~d}$. To examine effects on differentiated myotubes, agents to be tested were added on day 3 of differentiation, after myotube formation had occurred, and then cultures were incubated for another 2 $3 \mathrm{~d}$. For analysis, to unambiguously identify myotubes, the cultures were fixed and immuno-stained with an antibody against Myosin Heavy Chain-2 protein (MYH2), as described below.

Immunostaining and fluorescence analysis Cells were stained for Myosin Heavy Chain-2 protein (MYH2) as follows. Cells were fixed with $10 \%$ formalin for $20 \mathrm{~min}$ at room temperature (RT). Following 2-3 rinses with PBS, cells were permeabilized with $0.5 \%$ Triton X-100 (Sigma) in PBS for 15 min at RT. Non-specific binding was blocked with $2 \%$ BSA (Sigma), $0.25 \%$ Triton X-100 in PBS at RT for 30 min. Cells were then incubated with anti-skeletal myosin FAST primary antibody, MY-32 (Sigma), diluted 1:200 in blocking buffer for $2 \mathrm{~h}$ at RT. After rinsing with blocking buffer without Triton 2 times for $10 \mathrm{~min}$ each, cells were incubated with Alexa 488 (green) or Alexa 555 (red) conjugated anti-Mouse IgG secondary antibodies (both from Invitrogen) at 1:400 dilution along with Hoechst nuclear stain (\#33342; Sigma) at 1:5,000 dilution in blocking buffer at RT for $1 \mathrm{~h}$. Finally, cells were washed with PBS 3 times, $5 \mathrm{~min}$ each, before mounting with Vectashield containing Hoechst stain. All imaging was performed with a Nikon Eclipse Ti-S. Images were processed with NIS-Elements BR 3.10 software (Micro Video Instruments, Avon, MA). For quantitative analysis of immuno-stained samples, total myotube area was calculated as described in Semsarian et al. (1999) and Lecomte et al. (2010). Briefly, 5 images were captured from each of 3 replicate wells for each treatment group, for a total of 15 images per condition. A fluorescence intensity threshold was set such that only the MYH2-positive areas were measured 
by the software. The mean area for the untreated group (media only) was used to calculate the percent increase or decrease in myotube area for each treatment. This method allows a relatively rapid assessment of a compound's effect on myotube size.

Western blot analysis For Western blot analyses, antibodies against phosphorylated-Smad2 (ser465/467) (pSmad2), Smad2, phosphorylated-Akt (ser473) (pAKT), Akt, and alpha-tubulin were purchased from Cell Signaling Technologies (Danvers, MA). They were detected with peroxidaselabeled goat anti-rabbit IgG from Pierce (Rockford, IL).

Cells were washed in ice cold PBS before lysis in a buffer comprised of $40 \mathrm{mM}$ MOPS pH 7, $4 \mathrm{mM}$ EGTA, $10 \mathrm{mM}$ EDTA with 2\% Triton X-100, containing protease and phosphatase inhibitors $(30 \mathrm{mM}$ sodium fluoride, $60 \mathrm{mM} \beta$ glycerophosphate, $20 \mathrm{mM}$ sodium pyrophosphate, $1 \mathrm{mM}$ sodium orthovanadate, and $1 \%$ protease inhibitor cocktail, plus $1 \mathrm{mM}$ phenylmethylsulfonylfluoride (PMSF), all from Sigma. Cell lysates were centrifuged at $15,000 \mathrm{rpm}$ for $10 \mathrm{~min}$ at $4^{\circ} \mathrm{C}$. Supernatants were collected and protein contents were measured using the DC protein assay from Biorad (Hercules, CA). Samples were diluted in SDS-PAGE sample buffer and denatured for $5 \mathrm{~min}$ at $95^{\circ} \mathrm{C}$. Equal amounts of protein were loaded per lane on $10 \%$ Bis-Tris for NuPAGE gels, Invitrogen, electrophoresed, and then transferred on to nitrocellulose membranes. Membranes were blocked in Tris-buffered saline with $0.1 \%$ Tween -20 (TBS-T) with $5 \%$ (wt $/ \mathrm{vol}$ ) non-fat milk powder. Primary antibodies were diluted in TBS-T with 5\% BSA and secondary antibodies were diluted in TBS-T with 5\% non-fat milk. Immuno-reactivity was detected by SuperSignal West Pico Chemiluminescent substrate (Thermo Scientific, Rockford, IL) and exposed to film.

RNA analysis RNA was isolated using an RNeasy kit (Qiagen, Valencia, CA), following the manufacturer's protocol.

Quantitative real-time PCR (RT-PCR) was performed using an Applied Biosystems 7900 HT Fast Real-Time PCR System. The human Taqman probe sets for myosin heavy chain, MYH2 (Hs00430042_m1), Troponin T type 1, TNNT (Hs00162848_m1), myocyte enhancer factor 2C, Mef2C (Hs00231149_m1), myogenic differentiation 1, MYOD (Hs00159528_m1), myogenin, MYOG (Hs01072232_m1), and beta-2 microglobulin, B2M (\#4333766F), were purchased from Applied Biosystems (Wilmington, DE).

Adenoviral infection An adenovirus expressing GFP (adenoGFP) was purchased from ViraQuest (North Liberty, IA) (VQAd CMV eGFP titer: $5.0 \times 10^{10} \mathrm{PFU} / \mathrm{mL}$ ). For infection of myotubes, undifferentiated cells were plated at 20,000cells $/ \mathrm{cm}^{2}$ into 96 -well polystyrene cell culture plates and incubated overnight in growth medium and then switched to differentiation medium. After myotubes appeared (2-3 d), infection with adeno-GFP was performed. Several different ratios of viral particles to cells were tested (Multiplicity of Infection, or MOI, of 100, 250, 500, 700, and 1,000). Infections were performed in growth medium and incubated with the cells overnight $(18 \mathrm{~h})$. The following morning, this medium was removed and replaced with fresh growth medium and the cells were cultured for an additional $6 \mathrm{~h}$ before switching back to differentiation medium for $48 \mathrm{~h}$. The cells were then fixed for immuno-staining and visualization of GFP.

For infection of undifferentiated cells, the cells were plated at 20,000 cells $/ \mathrm{cm}^{2}$ in 96 -well tissue culture plates and incubated for $24 \mathrm{~h}$ in growth medium. For infection, the same MOIs and procedure described above were utilized. In some cases, to observe the effects of infection on myotube differentiation, cells were returned to differentiation medium for $6 \mathrm{~d}$ after a $6 \mathrm{~h}$ recovery period in growth medium.

Statistical analysis Results are expressed as means \pm standard deviations. All data analysis was performed using Microsoft Excel 2007. Comparisons between two groups were performed using an unpaired Student's $t$ test. All tests were considered significant when $p<0.05$.

\section{Results}

Primary human skeletal muscle myoblasts differentiate into multinucleated myotubes in culture Four different primary human muscle cell preparations from three different commercial vendors were screened for the ability to differentiate into multinucleated myotubes in cell culture. We tested human skeletal muscle myoblasts (HSMM; Lonza), human skeletal muscle cells (SkMC-L; Lonza), human skeletal muscle-derived cells (SkMDC; Cook Myosite), and human skeletal muscle cells (SkMC-P; PromoCell). Initial experiments found that two of these preparations (SkMC-L and SkMC-P) showed little, if any, evidence of morphological differentiation into myotubes when cultured according to the recommendations of the suppliers. In addition, SkMC-P grew very poorly as undifferentiated cells in growth medium. However, HSMM and SkMDC demonstrated robust myotube differentiation, and could be readily cultured as undifferentiated cells in growth medium, using polystyrene cell culture plates. Thus, we focused on these two primary cell preparations for the remainder of our studies presented here. HSMM were provided as a stock of myoblasts cryopreserved at passage 2 and characterized by the vendor to display greater than $60 \%$ desmin-positive cells at first passage out of cryopreservation. SkMDC were characterized as displaying 92\% desmin-positive cells and a gene expression analysis provided by the vendor showed that these cells expressed several markers of muscle cell precursors such as Pax3, Pax7, 
MYOD1, MYOG, and Myf5. Also SkMDC did not express MYH2 as myoblast cultures. This marker analysis therefore suggests that the SkMDC population is a committed myoblast cell population. For both HSMM and SkMDC, the vendors recommend that these cell populations should be used for experiments at a population doubling (PD) less than 10. In our initial studies, we evaluated cell growth and differentiation of cells through 15 PDs. SkMDC had a PD time of $24 \mathrm{~h}$ for up to 4-5 passages, after which their doubling time slowed gradually until 15 PDs were reached. HSMM were slower to recover from cryopreservation compared to SKMDC. For the first $5 \mathrm{~d}$, their PD time was approximately $40 \mathrm{~h}$, after which it decreased to $24 \mathrm{~h}$ for about $7 \mathrm{~d}$ and then increased to $48 \mathrm{~h}$ up to 15 PDs. In general, above 10 PDs, both cell types took longer to differentiate and their differentiation was not as robust, compared to cells at $\mathrm{PD}<10$ (data not shown). For the studies described in this paper, we used cells that had undergone no more than 7 PD. Cells were also plated on several different extracellular matrix protein (ECM)-coated culture plates to assess their effect on cell growth and differentiation. The following human ECMs were screened using Millipore's Millicoat ECM-coated strips: fibronectin, vitronectin, laminin, collagen type I, and collagen type IV. In addition, we also screened BD PureCoat Amine (BD Biosciences, San Jose, $\mathrm{CA}$ ) and polystyrene plates coated in-house with $2 \%$ gelatin (Sigma). No obvious improvement in cell growth or differentiation was detected with any of these matrices compared to regular tissue-culture treated polystyrene plates. Therefore, standard cell-culture grade polystyrene plates were used throughout these studies.

Both HSMM and SkMDC differentiated into myosin heavy chain (MYH2)-positive multinucleated myotubes within $3 \mathrm{~d}$ when cultured on polystyrene cell culture plates in differentiation medium (Fig. 1A). In general, the undifferentiated cells cultured in growth medium did not express MYH2, although occasional MYH2-positive cells were seen in growing cultures, possibly resulting from cell confluence associated contact-dependent cell differentiation (see SkMDC in Fig. 1A). To minimize this issue, care was taken to subculture growing cultures of cells before they became confluent. It was noted that the organization of nuclei within the myotubes was different between HSMM and SkMDC. In HSMM myotubes, the nuclei tend to be arranged as singlets, or small groups, in linear arrays. In contrast, the nuclei in SkMDC myotubes were often clustered together in large groups (Fig. 1A). This difference may be caused by differences in the mechanisms of cell fusion during myotube generation, or by differences in the regulation of nuclear dynamics in the myotubes, or other unknown factors. To further characterize the differentiation process of HSMM and SkMDC, RNA was prepared from cells harvested on day 0 through day 6 of culture in differentiation medium. Gene expression analyses were performed using quantitative
RT-PCR for various markers of muscle cell differentiation, (Fig. 1B). In all cases, gene expression levels were normalized to beta-2 microglobulin (B2M) expression levels in order to assess changes in relative expression. MyoD and $\mathrm{Mef} 2 \mathrm{C}$, transcription factors that play important roles in specifying the myogenic lineage, represent two early markers of myoblast differentiation into myotubes (Megeney and Rudnicki 1995; Black and Olson 1998; Zammit et al. 2006). In both HSMM and SkMDC, there was a transient, modest upregulation of MyoD at day 1 (although it was statistically significant for HSMM only) which returned to baseline levels by day 2. Mef2C was also upregulated by day 1 in both cell populations, reaching statistical significance by day 2, but unlike MyoD, the expression of this gene remained elevated throughout the 6-d culture period. Myogenin (MYOG), Troponin T (TNNT), and MYH2 represent late markers of muscle cell differentiation (Burattini et al. 2004; Ozernyuk and Balan 2007). Similar expression trends with these markers were seen in HSMM and SkMDC cultures. MYOG was upregulated approximately tenfold by day 1 of differentiation and that expression level remained significantly elevated from day 2 to day 6 of culture. TNNT was significantly upregulated by day 1 of differentiation in HSMM and by day 2 of differentiation for SkMDC. TNNT expression remained at least tenfold upregulated through day 6 of differentiation in both cell populations. MYH2 was also expressed at least fivefold above control expression levels by day 1 of differentiation for HSMM and by day 2 of differentiation for SkMDC. Then, MYH2 expression reached greater than tenfold above control levels through day 6 of differentiation. Thus, HSMM and SkMDC undergo morphological differentiation in culture into multinucleated myotubes and express characteristic molecular markers of muscle cell differentiation.

Induction of morphological atrophy and hypertrophy Dexamethasone, a synthetic steroid, and myostatin, a growth factor which is a negative regulator of muscle mass, are well-characterized inducers of myotube atrophy, as shown for C2C12 myotubes (Lee 2004; Stitt et al. 2004). Also, C2C12 myotubes display a hypertrophic response following treatment with insulin-like growth factor-1 (IGF-1; Semsarian et al. 1999). Therefore, to examine the response of primary human skeletal muscle cell cultures to these factors, myotubes derived from HSMM and SkMDC were treated with various concentrations of Dex, Mstn, or IGF-1 for $48 \mathrm{~h}$ and then fixed and immuno-stained for MYH2. Nuclei were visualized by Hoechst-staining. Representative images of untreated myotubes or those treated with Mstn $(1 \mu \mathrm{g} / \mathrm{ml})$ or Dex $(50 \mu \mathrm{M})$ are shown in Fig. $2 A$ and with IGF-1 $(1 \mu \mathrm{g} / \mathrm{ml})$ are shown in Fig. 2B. HSMM and SkMDC undergo an atrophy-like response to both Mstn and Dex treatment, while only HSMM display a hypertrophy-like 
A

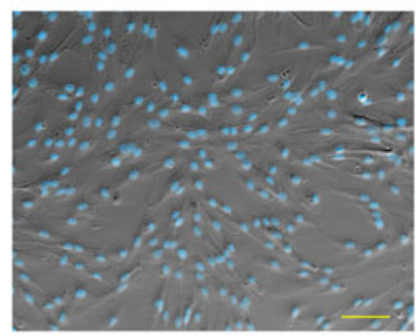

B

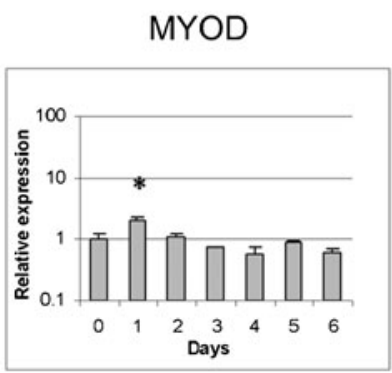

MYOG

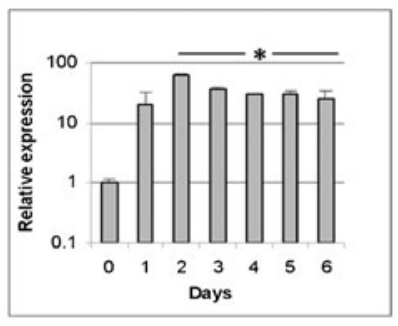

HSMM

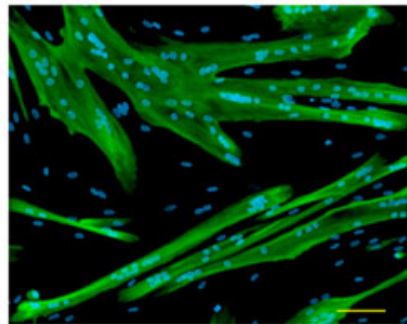

Mef2C

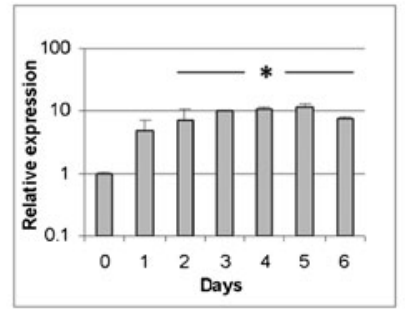

TNNT

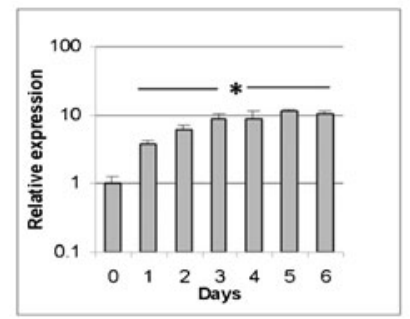

MYH2

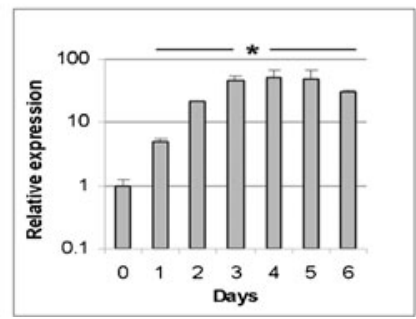

Figure 1. HSMM and SkMDC differentiated into multinucleated Myosin Heavy Chain-2 (MYH2)-positive myotubes. (A) Representative phase contrast and dark-field images of MYH2-immunostained cells (green), with Hoechst-labeled nuclei (blue) after $1 \mathrm{~d}$ in growth medium (phase contrast images) or $3 \mathrm{~d}$ in differentiation medium (dark-field images; magnification $\times 10$; scale bar $=100$ microns). (B) Skeletal

response to IGF-1. Total myotube areas were measured as described in Semsarian et al. (1999) and Lecomte et al. (2010) and results were plotted as the percent decrease or increase in myotube area compared to untreated control cultures (Fig. 2A, B). The plots show that Mstn and Dex induced statistically significant decreases in myotube area over untreated control cultures at all concentrations (except for the HSMM $250 \mathrm{ng} / \mathrm{ml}$ Mstn-treated group which showed decreased myotube area but failed to reach statistical

SkMDC

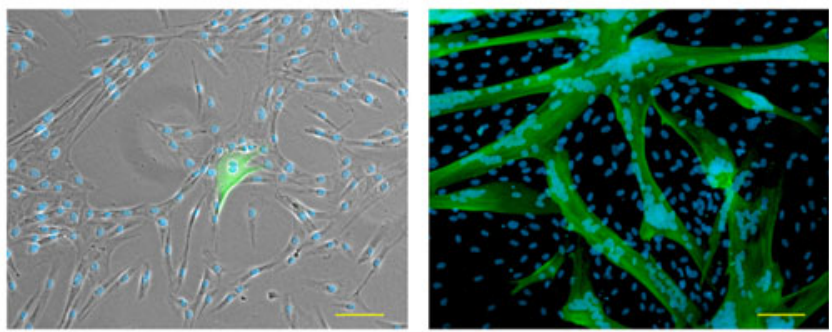

SkMDC

MYOD

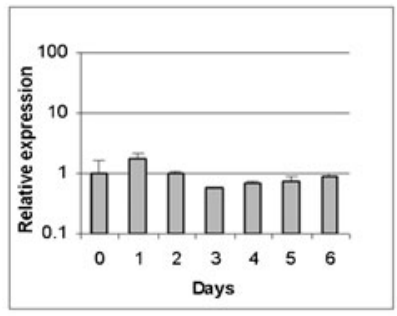

MYOG

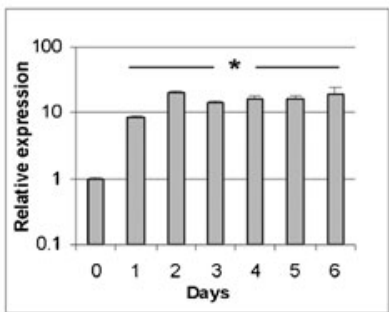

Mef2C

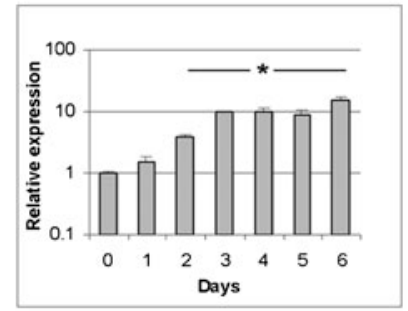

TNNT

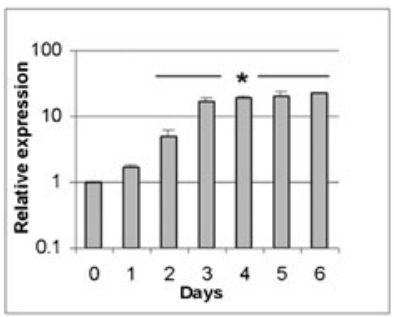

MYH2

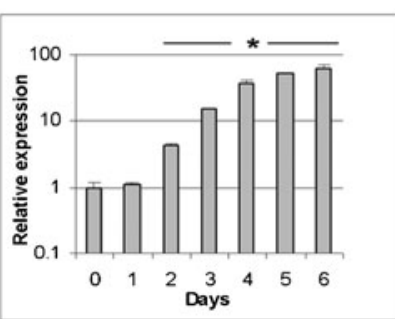

muscle cell marker gene expression by RT-PCR. Plots show gene expression of early (MYOD, Mef2C) and late (MYOG, TNNT, and MYH2) muscle cell markers, normalized to B2M gene expression, relative to expression levels at day 0 (time at which differentiation media was added to the cells); ${ }^{*} p<0.05$ vs. day 0 using Student's $t$ test.

significance; Fig. 2A). Quantification of IGF-1 effects on both cell populations confirmed that only HSMM cultures responded with a hypertrophic response, with significant increases in myotube area at 100 and $1,000 \mathrm{ng} / \mathrm{ml} \mathrm{IGF-1,}$ (Fig. 2C). Statistical significance, using Student's $t$ test was performed by comparing the total myotube area in multiple untreated cultures with the total myotube area in treated cultures and then assigning statistical significance $(*)$ if $p<0.05$. 
A
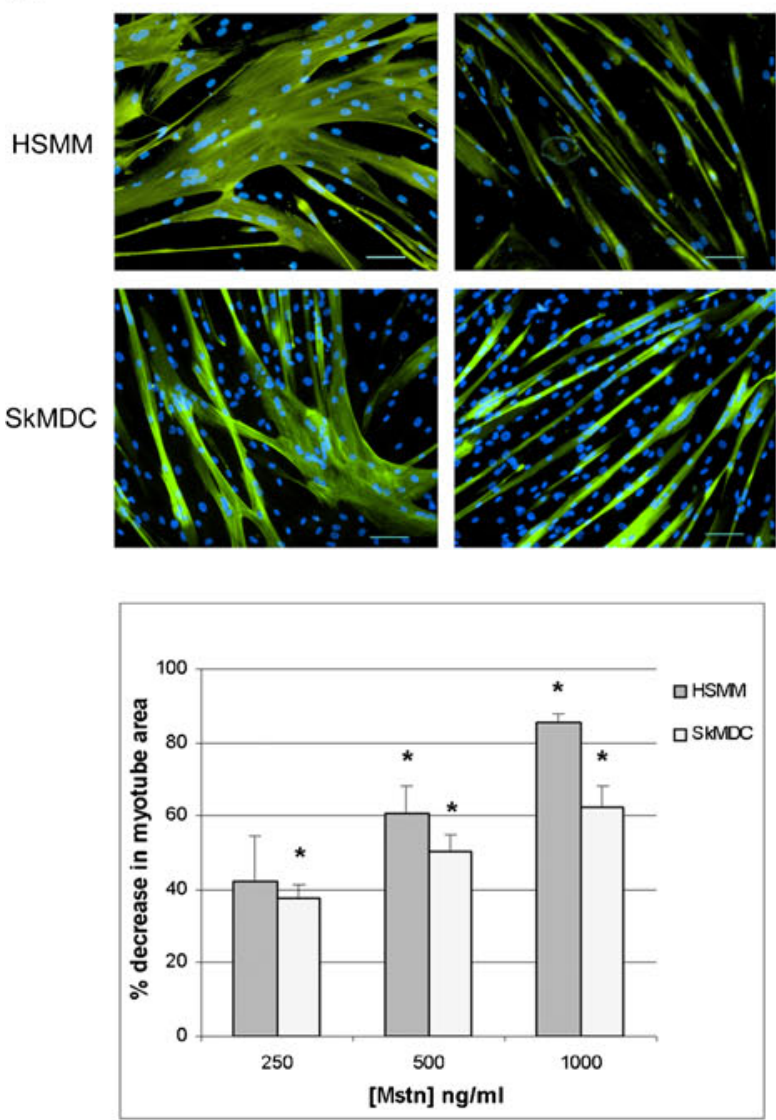

B

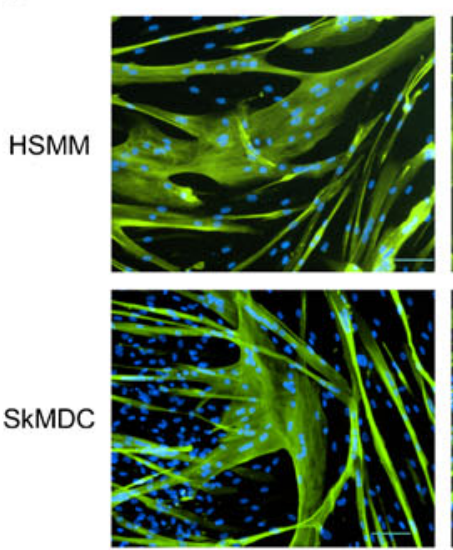

C
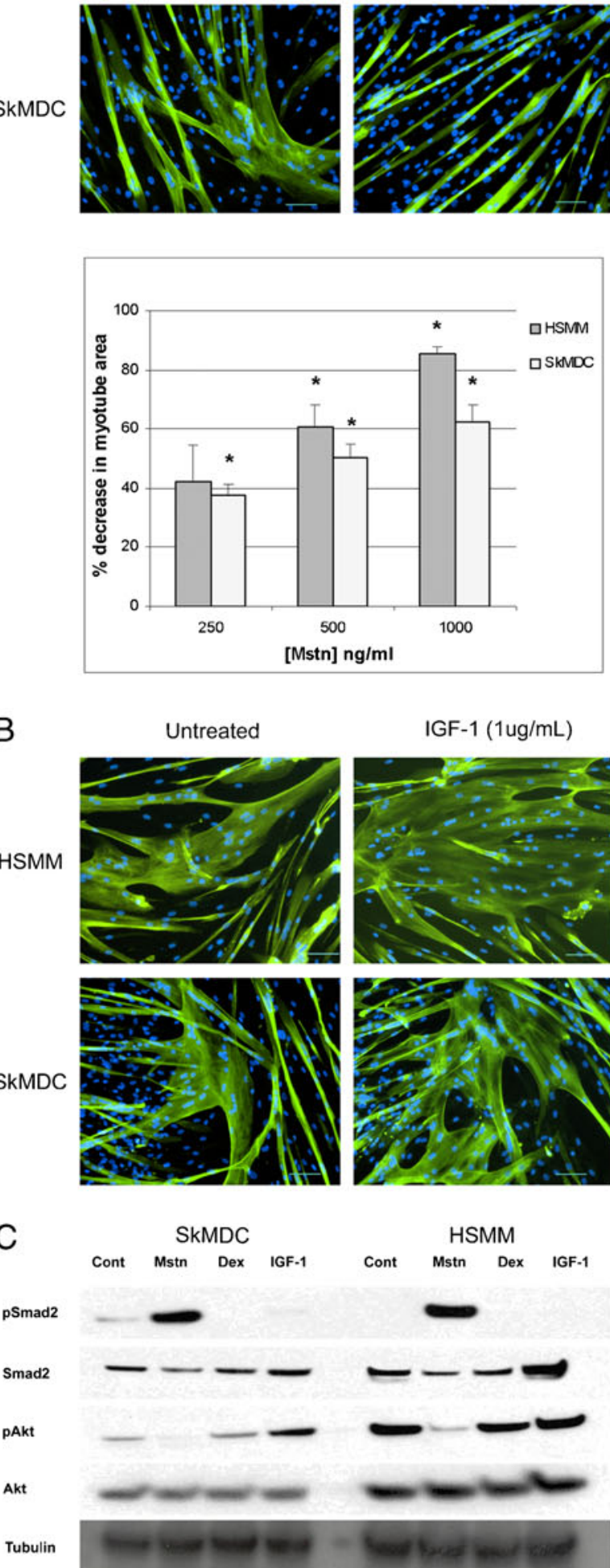

GF-1 (1ug/mL)
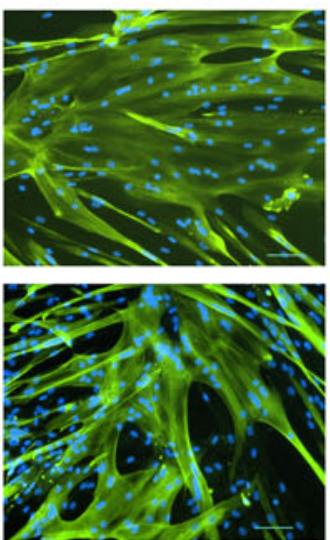

Untreated
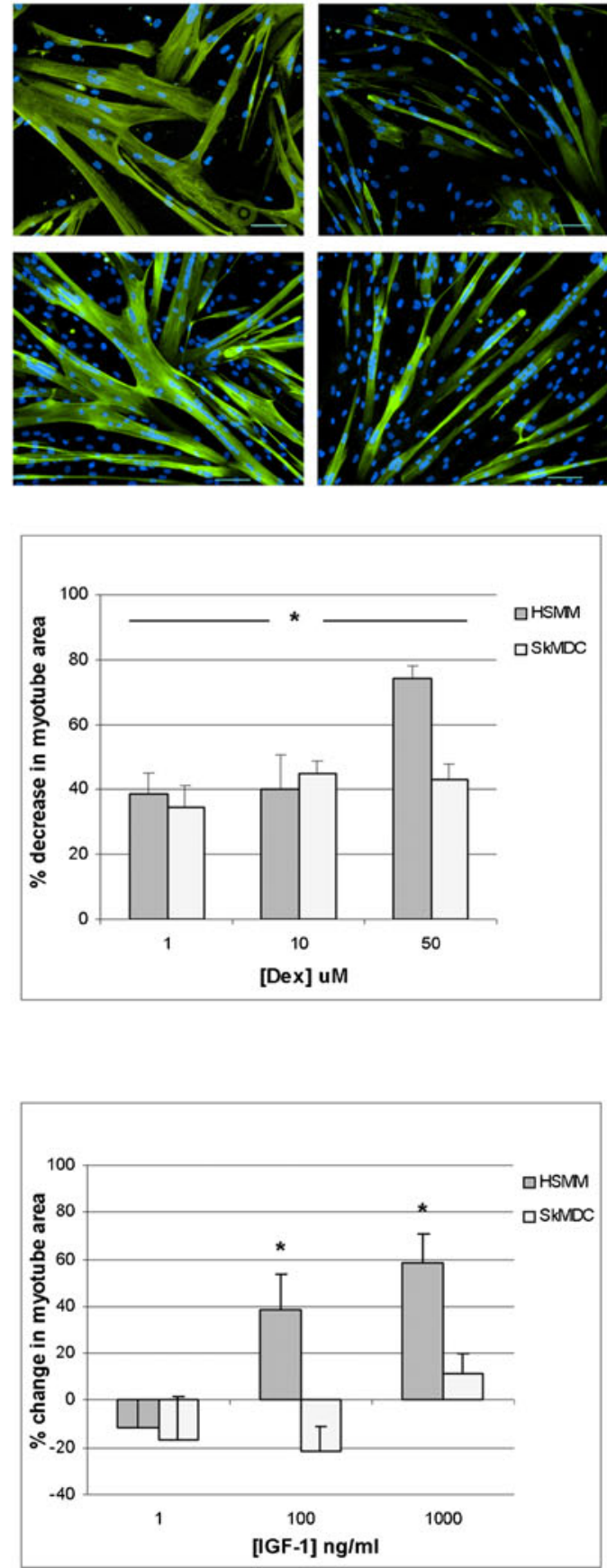
4 Figure 2. Induction of atrophy and hypertrophy in HSMM and SkMDC myotubes. (A) Differentiated myotubes were treated with a concentration series of Mstn or Dex for $48 \mathrm{~h}$. Representative images of MYH2/Hoechst-labeled myotubes treated with $1 \mu \mathrm{g} / \mathrm{ml} \mathrm{Mstn} \mathrm{or} 50 \mu \mathrm{M}$ Dex for $48 \mathrm{~h}$. Plots of the percent decrease, from untreated cells, in myotube area at each concentration of test agent are shown. (All plots represent means \pm SEM, $n=15 .{ }^{*} p<0.05$ vs. untreated using Student's $t$ test. All images at $\times 10$ magnification; scale bar $=100$ microns). $(B)$ Differentiated myotubes were treated with a concentration series of IGF-1 for $48 \mathrm{~h}$. Representative images of $\mathrm{MYH} / \mathrm{Hoechst}$-labeled myotubes following treatment with $1 \mu \mathrm{g} / \mathrm{ml}$ insulin-like growth factor-1 (IGF-1). Plots show the percent increase, from untreated cultures, in myotube area at each concentration of IGF-1. (All plots represent means \pm SEM, $n=15$. ${ }^{*} p<0.05$ vs. untreated using Student's $t$ test. All images at $\times 10$ magnification; scale bar $=100$ microns). (C) Western blots of myotube lysates from cultures treated with $1 \mu \mathrm{g} / \mathrm{ml}$ Mstn, $50 \mu \mathrm{M}$ Dex, or $1 \mu \mathrm{g} / \mathrm{ml} \mathrm{IGF-1} \mathrm{for} 48 \mathrm{~h}$ and probed with antibodies to phosphorylated- and total Smad2 and phosphorylatedand total AKT. Anti-alpha tubulin was used as a loading control.

To examine the signaling pathways that were activated during these treatments, cell lysates were prepared at the end of the $48 \mathrm{~h}$ treatment period and then probed with antibodies to phosphorylated- and total Smad2, and phosphorylatedand total AKT, since these molecules are involved in the earliest stage responses to the Mstn and IGF-1 ligands (Rebbapragada et al. 2003; Duan et al. 2010). Upon binding to cell surface receptors, the myostatin-receptor complex directly phosphorylates the signaling molecules Smad2 and Smad3, Rebbrapragada et al. (2003). In both HSMM and SkMDC, Mstn treatment increased phosphorylated-Smad2 (pSmad2) levels (Fig. 2C) consistent with this ligand's known signaling activity described above. In addition, Mstn reduced the level of phosphorylated-AKT (pAKT) in HSMM and SkMDC (Fig. 2C). This effect has also been observed in C2C12 myotubes (McFarlane et al. 2006). Inhibition of AKT phosphorylation is consistent with an atrophic response, since pAKT activity is a known positive regulator of muscle protein synthesis and growth (Duan et al. 2010). In contrast to myostatin, Dex treatment induced downregulation of the control levels of pSmad2 in SkMDC (Fig. 2C). pSmad2 was not detectable in untreated HSMM cultures, so it is unclear if Dex affected pSmad2 levels in these cultures. In our studies, Dex did not affect pAKT levels in either cell population.

Overall, these results suggest that at least for SkMDC, Mstn and Dex operate through separate pathways to induce an atrophy-like response. Finally, IGF-1 binding to its receptor is known to stimulate the phosphorylation of AKT, which stimulates protein synthesis and cell growth (Duan et al. 2010). IGF1 treatment of SkMDC, but not HSMM, increased pAKT levels (Fig. 2C). These results are puzzling since only HSMM, and not SkMDC, showed a morphological response to IGF-1, with a substantial increase in myotube area (Fig. $2 B$ ). This could be due to the relatively high pAKT levels in untreated HSMM compared to SkMDC which may help prime the cells' hypertrophic response to exogenous IGF-1. It is also possible that
IGF-1 acts through an AKT-independent mechanism to induce a hypertrophy-like response in HSMM and is insufficient to cause such a response in SkMDC.

In addition to Mstn and Dex, the cytokine TNF-related weak inducer of apoptosis (TWEAK) has been reported to be a potent inducer of muscle atrophy (Dogra et al. 2007a) and blocks the differentiation of $\mathrm{C} 2 \mathrm{C} 12$ myoblasts into myotubes (Dogra et al. 2006, 2007b). In our studies, treatment of HSMM and SkMDC myotubes with TWEAK had no observable effects (data not shown). However, when added to the culture at the start of myotube differentiation, the differentiation of SkMDC was completely blocked, while it had no effect on HSMM differentiation (Fig. 3). Expression of the TWEAK receptor, FN14, has been demonstrated on human muscle satellite cells (Girgenrath et al. 2006), but it is possible that FN14 is not expressed by HSMM which would explain the absence of an effect of TWEAK in these cultures. We examined FN14 expression in both cell populations by real-time RT-PCR and found that it was expressed in both myoblasts and myotubes and showed no changes during differentiation (data not shown).

Rescue of myostatin-induced atrophy As shown above, Mstn induced an atrophy-like morphological response in both HSMM and SkMDC where the myotubes showed a substantial decrease in size compared to untreated cultures (Fig. 2A). We and others are working to target myostatin with inhibitors to block its negative regulation of muscle mass as a treatment for frailty. One such inhibitor consists of a fusion between the ligand-binding domain of ActRIIb, the high affinity receptor for Mstn, and the Fc region of immunoglobulin G (ActRIIb-Fc). This molecule is a potent inhibitor of Mstn activity in vivo (Lee et al. 2005). To assess its activity in

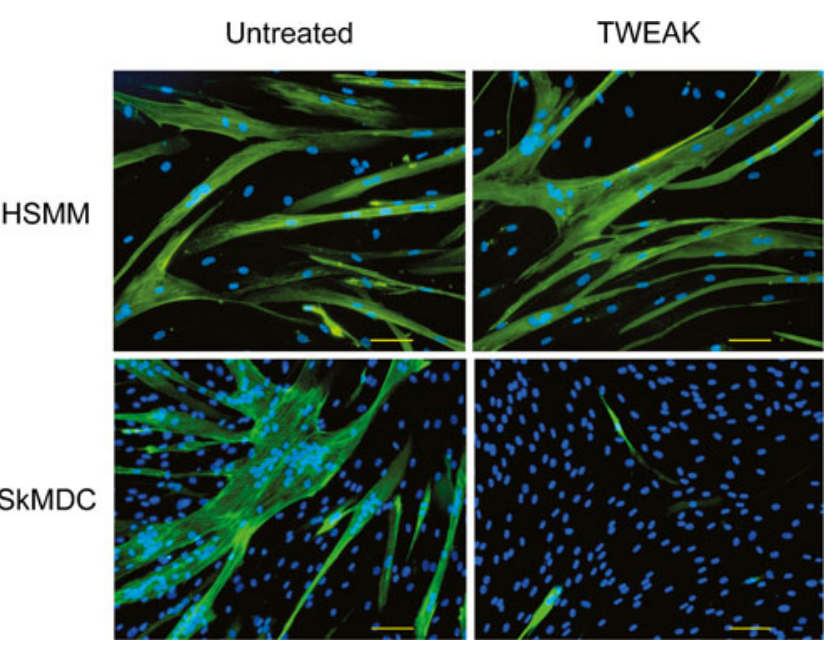

Figure 3. Effect of TWEAK on HSMM and SkMDC. Representative images of $\mathrm{MYH} 2 / \mathrm{Hoechst}-$ labeled myotubes from cultures differentiated for $4 \mathrm{~d}$ in differentiation medium with TWEAK $(1 \mu \mathrm{g} / \mathrm{ml})$. (Magnification $\times 10$; scale bar $=100$ microns). 
HSMM and SkMDC, myotubes were treated with Mstn in the presence of various concentrations of ActRIIb-Fc for $48 \mathrm{~h}$. Images of MYH2-positive myotubes were captured and myotube areas were quantified as described above. As expected, the images showed that Mstn alone induced a significant decrease in myotube area, and this atrophy-like response was blocked by ActRIIb-Fc in both cell populations (Fig. 4). The rescue of Mstn-induced atrophy by ActRIIb-Fc was quantified and plotted as the percent change from untreated myotubes (Fig. 4). In both cell populations, $50 \mu \mathrm{g} / \mathrm{ml}$ ActRIlb-Fc induced a significant percent change in area compared to cells treated with Mstn $(1 \mu \mathrm{g} / \mathrm{ml})$ alone $(0 \mu \mathrm{g} / \mathrm{ml}$ ActRIIB-Fc). The plots demonstrated that ActRIIB-Fc blocked Mstn-induced atrophy in these cultures, such that the myotube area was significantly different to that measured in cultures treated with Mstn alone. At lower concentrations of ActRIIb-Fc, statistically significant effects were shown in SkMDC cultures only, suggesting that these cells were more sensitive to the rescue effect. Thus, HSMM and SkMDC may be used to identify and characterize inhibitors of myostatin activity.

Gene transfer into HSMM and SkMDC The ability to genetically manipulate cells in culture provides powerful tools for analysis. Hence, various methods of introducing DNA into HSMM and SkMDC were investigated. Transfection of both growing cells as well as differentiated myotubes was attempted. Lipid-mediated transfection reagents were largely
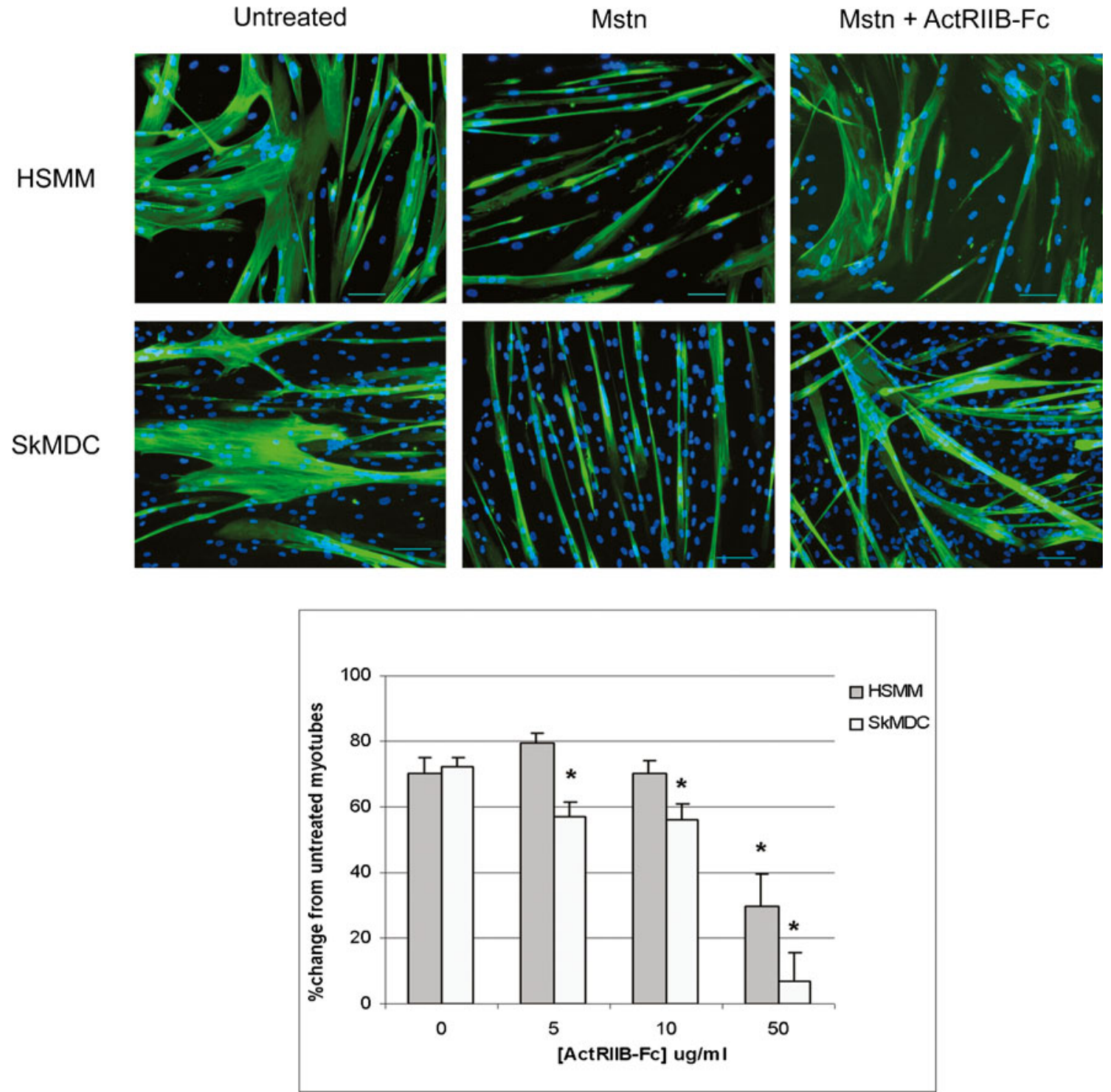

Figure 4. ActRIIB-Fc (a myostatin inhibitor) blocks myostatin-induced atrophy in HSMM and SkMDC. MYH2/Hoechst-labeled myotubes differentiated for $3 \mathrm{~d}$ and then treated with $1 \mu \mathrm{g} / \mathrm{ml}$ myostatin \pm a dilution series of ActRIIB-Fc for $48 \mathrm{~h}$. Representative images of untreated myotubes and those treated with $1 \mu \mathrm{g} / \mathrm{ml} \mathrm{Mstn}$ alone and $1 \mu \mathrm{g} / \mathrm{ml} \mathrm{Mstn}+50 \mu \mathrm{g} / \mathrm{ml}$ ActRIIB-Fc. Plot shows the mean percent change in myotube area from untreated myotubes \pm SEM, $n=15$, ${ }^{*} p<0.05$ vs $1 \mu \mathrm{g} / \mathrm{ml}$ Mstn using Student's $t$ test). 
ineffective (we estimated that $<20 \%$ of the cells were transfected as assessed by the number of GFP-positive cells), although HSMM appeared to be slightly more susceptible to transfection by this method than SkMDC (data not shown). In addition, we observed that most lipid transfection reagents significantly slowed down the differentiation of myotubes, requiring $6 \mathrm{~d}$ for full differentiation compared to 2-3 d for untreated cultures (data not shown). Similar results were obtained using electroporation methods with the undifferentiated cells (data not shown).

To investigate an alternative method of gene delivery to HSMM and SkMDC, an adenovirus expressing GFP was used to infect differentiated myotubes. Myotubes were identified by staining with anti-MYH2 and detected with Alexa Fluor 555 goat antimouse IgG secondary antibody (red), while the infected cells were detected by GFP fluorescence (green). Infected myotubes were orange (red plus green), uninfected myotubes were red, and infected, undifferentiated cells were green. Although not quantified, it was noted that almost all the myotubes were orange. In addition, most of the undifferentiated (nonmyotube) cells in the cultures were also successfully infected, displaying mostly GFP-positive cells (Fig. 5). Finally, undifferentiated cells were also effectively infected by the adenovirus, although the infection efficiency varied from experiment to experiment from $\sim 30 \%$ to $80 \%$, quantified by visual inspection only (data not shown). However, infection of the undifferentiated cells slowed down myotube differentiation by about 3-4 d (data not shown). In general, adenoviral infection was found to be the most efficient method for gene delivery into both cell populations.

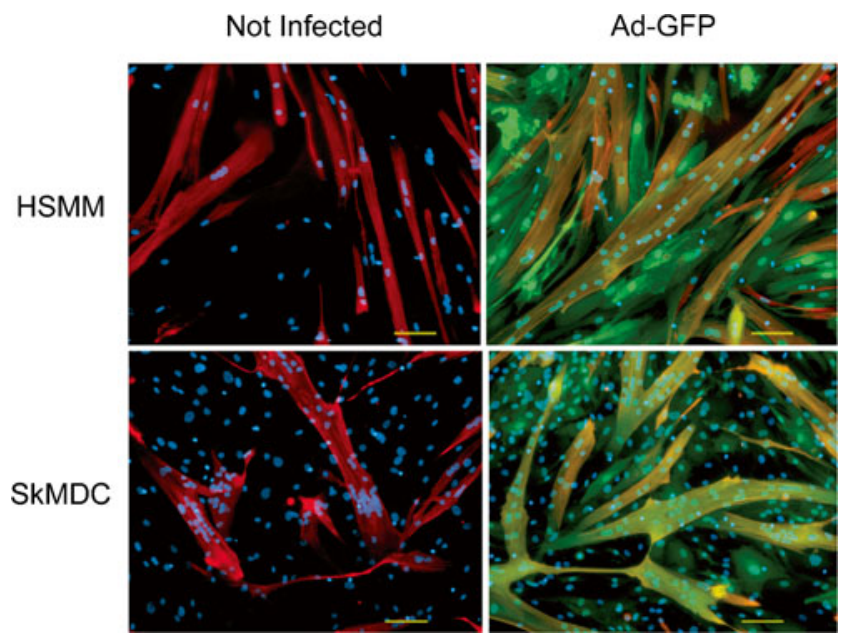

Figure 5. Infection of HSMM and SkMDC with adenovirus. Representative images of MYH2/Hoechst-labeled myotubes differentiated for $2 \mathrm{~d}$ and then infected with adeno-GFP at 500 MOI. MYH2 stained red (Not infected images) in this experiment and adeno-GFP is green. The overlap results in a yellow/orange color which demonstrates expression of GFP in MYH2-positive myotubes (Ad-GFP images). (Magnification $\times 10$; scale bar $=100$ microns).

\section{Discussion}

These studies were performed to screen several commercially available sources of primary human skeletal myoblasts for their capacity to reproducibly differentiate into myotubes and respond appropriately to inducers of atrophy and hypertrophy. Out of the four cell types that were screened, only two demonstrated these features (HSMM from Lonza and SkMDC from Cook Myosite). Although HSMM and SkMDC both differentiate into myotubes, we observed several marked differences between them. First, for HSMM there were notably fewer cell nuclei that were not incorporated into myotubes after culturing in differentiation medium compared to SkMDC (see darkfield images in Fig. 1). This observation could be explained by a number of factors. For example, although both cell populations were plated at the same density, differences in the rate of cell proliferation prior to the induction of differentiation could be responsible, as could different myoblast fusion efficiencies. Alternatively, greater cell death during the 3-d differentiation period in the HSMM cultures could cause this effect, or the unincorporated nuclei may represent another cell type that the SkMDC can generate during differentiation. Second, as noted in the results, the organization of nuclei within the myotubes was different between HSMM and SkMDC. Third, HSMM and SkMDC showed different responses to some extrinsic factors. For example, SkMDC did not undergo a morphological hypertrophy response when treated with IGF-1 while HSMM showed distinct increases in myotube size. We are currently unable to explain this insensitivity to IGF-1 shown by SkMDC. Furthermore, we observed that SkMDC were more sensitive to higher concentrations of Dex. These cells die when exposed to $100 \mu \mathrm{M}$ Dex whereas HSMM do not (data not shown). The finding that Dex had no effect on pAKT levels in either cell population (see Fig. 2C) was surprising because it has been shown that Dex treatment reduced pAKT levels in C2C12 myotubes (Zhao et al. 2009). However, in those experiments, C2C12 myotubes were treated with only $100 \mathrm{nM}$ Dex overnight. In our studies, myotubes were treated with $50 \mu \mathrm{M}$ Dex for $48 \mathrm{~h}$. Such culture conditions induced visible and quantifiable myotube atrophy, whereas $100 \mathrm{nM}$ Dex failed to induce a visible atrophy. It is possible that the absence of Dex-regulated pAKT levels in our studies may be due to the higher Dex concentration and longer incubation time compared to previous studies with $\mathrm{C} 2 \mathrm{C} 12$ myotubes, or that this is a novel feature of primary human skeletal muscle cells.

Finally, the cytokine TWEAK inhibited the differentiation of SkMDC but not HSMM (Fig. 3) and had no effect on differentiated SkMDC and HSMM myotubes (data not shown). TWEAK has been shown to induce atrophy in differentiated C2C12 myotubes (Dogra et al. 2007a) and prevent the differentiation of $\mathrm{C} 2 \mathrm{C} 12$ myoblasts into myotubes (Dogra et al. 2006, 2007b). However, we are not aware that similar effects of TWEAK on primary human skeletal muscle 
cells have been examined. Our findings with TWEAK were not explained by the absence of the appropriate TWEAK receptor (FN14) on these cells, both as myoblasts and myotubes (data not shown). Therefore, we are unable to explain the absence of an effect of TWEAK on the differentiation of HSMM and the absence of TWEAK-induced atrophy in the myotubes derived from both cell populations at this time. It is possible that all of these differences may be due to the origins of the cells. HSMM were derived from the quadriceps muscle of a young adult male (16 yr old) cadaver, while SkMDC came from the rectus abdominus muscle of an older male ( $52 \mathrm{yr}$ old) living donor. It is possible, even likely, that the precise characteristics of primary human skeletal muscle cells will be affected by numerous donor factors, such as age, gender, and general health. Thus, comparison of results from experiments using cells from different donors may be challenging.

During our analyses, we noticed differences in the gene expression profile for MYOD and MYOG in both cell populations compared to what has been published for primary skeletal muscle cells (Cornelison and Wold 1997; Zammit et al. 2006). In this work, it was shown that MYOD expression is typical of a transcription factor with a rapid significant increase in expression upon commitment of satellite cells to the muscle lineage. This expression is then followed by rapid downregulation of expression, at the same time as MYOG expression is turned on. In our studies, we failed to demonstrate a significant upregulation of MYOD after $24 \mathrm{~h}$ of culture in differentiation medium. It is possible that MYOD was upregulated within $10 \mathrm{~h}$ and so we were measuring the down regulation of its expression at $24 \mathrm{~h}$. It is also possible that both cell populations represent committed muscle precursors, which already express MYOD, so the fold change in expression upon switching to differentiation medium would be minimal. Indeed, the SkMDC vendor (Cook Myosite) has shown that as myoblasts, these cells already express MYOD (Janowski 2011). In our cultures, MYOG expression was maximal between 24 and $48 \mathrm{~h}$ in differentiation medium. Expression of MYOG has been shown as early as $10 \mathrm{~h}$ after switching to differentiation medium, with highest expression levels seen before 48 and $60 \mathrm{~h}$ in differentiation medium (Bigot et al. 2008). It is possible that our cultures also upregulate MYOG expression at earlier time-points in differentiation medium, but we did not harvest RNA at time-points earlier than $24 \mathrm{~h}$. The prolonged expression profile of MYOG from the $24 \mathrm{~h}$ time-point in our cultures agrees with what has been shown for primary human muscle cells (Bigot et al. 2008).

In summary, we have identified two commercially available sources of primary human skeletal muscle cells that can reproducibly differentiate into multinucleated myotubes in culture. Myotube differentiation occurs rapidly upon shifting the cells into differentiation medium, within $2-3 \mathrm{~d}$, and they express characteristic molecular markers of muscle differentiation. The myotubes derived from these cells can be induced to undergo morphological atrophy- and hypertrophy-like responses. We have also shown that the atrophy response can be blocked with a pharmacological inhibitor of myostatin, a known negative regulator of muscle mass. Finally, these myotubes can be efficiently infected with adenoviruses, providing a method for genetic modification. Such modifications would potentially allow adenoviral-shRNA knock-down screens for muscle atrophy targets which could then be targeted for inhibition by small or large molecule compounds. Taken together, our results indicate that these primary human skeletal muscle cells may be a useful system for studying skeletal muscle cell differentiation and may also provide tools for studying new therapeutic molecules such as myostatin inhibitors.

Acknowledgments We thank Xianfeng Li, Ti Wang, Ru Ding, and Mark Johnson for their expert technical assistance.

Open Access This article is distributed under the terms of the Creative Commons Attribution License which permits any use, distribution, and reproduction in any medium, provided the original author(s) and the source are credited.

\section{References}

Baudy A.; Saxena H.; Gordish H.; Hoffman E.; Nagaraju K. A robust in vitro screening assay to identify NF-kappaB inhibitors for inflammatory muscle diseases. Int. Immunopharmacol. 9: 12091214; 2009.

Berger M.; Doherty T. Sarcopenia: prevalence, mechanisms and functional consequences. Interdiscip. Top. Gerontol. 37: 94-114; 2010.

Bigot A.; Jacquemin V.; Debacq-Chainiaux F.; Butler-Browne G.; Toussaint O.; Furling D.; Mouly V. Replicative aging downregulates the myogenic regulatory factors in human myoblasts. Biol. Cell 100: 189-199; 2008.

Black B.; Olson E. Transcriptional control of muscle development by myocyte enhancer factor-2 (MEF2) proteins. Annu. Rev. Cell Dev. Biol. 14: 167-196; 1998.

Burattini S.; Ferri P.; Battistelli M.; Curci P.; Luchetti F.; Falcieri E. $\mathrm{C} 2 \mathrm{C} 12$ murine myoblasts as a model of skeletal muscle development: morpho-functional characterization. Eur. J. Histochem. 48: 223-234; 2004.

Cho Y.; Kim C.; Cheon H. Cell-based assay for screening 11betahydroxysteroid dehydrogenase 1 inhibitors. Anal. Biochem. 392: 110-116; 2009.

Cornelison D.; Wold B. Single-cell analysis of regulatory gene expression in quiescent and activated mouse skeletal muscle satellite cells. Dev. Biol. 191: 270-283; 1997.

Cui Z.; Chen X.; Lu B.; Park S.; Xu T.; Xie Z.; Xue P.; Hou J.; Hang H.; Yates J.; Yang F. Preliminary quantitative profile of differential protein expression between rat L6 myoblasts and myotubes by stable isotope labeling with amino acids in cell culture. Proteomics 9: $1274-1292 ; 2009$.

Dogra C.; Changotra H.; Mohan S.; Kumar A. Tumor Necrosis Factor-like Weak Inducer of Apoptosis Inhibits Skeletal Myogenesis through Sustained Activation of Nuclear Factor- $\mathrm{kB}$ and Degradation of MyoD Protein. J. Biol. Chem. 281: 10327-10336; 2006. 
Dogra C.; Changotra H.; Wedhas N.; Qin X.; Wergedal J.; Kumar A. TNF-related weak inducer of apoptosis (TWEAK) is a potent skeletal muscle-wasting cytokine. FASEB J. 21: 1857-1869; 2007a.

Dogra C.; Hall S.; Wedhas N.; Linkhart T.; Kumar A. Fibroblast growth factor inducible 14 (Fn14) is required for the expression of myogenic regulatory factors and differentiation of myoblasts into myotubes. Evidence for TWEAK-independent functions of Fn14 during myogenesis. J. Biol. Chem. 282: 15000-15010; 2007b.

Duan C.; Ren H.; Gao S. Insulin-like growth factors (IGFs), IGF receptors and IGF-binding proteins: role in skeletal muscle growth and differentiation. Gen. Comp. Endocrinol. 167: 344-351; 2010.

Girgenrath M.; Weng S.; Kostek C.; Browning B.; Wang M.; Brown S.; Winkles J.; Michaelson J.; Allaire N.; Schneider P.; Scott M.; Hsu Y.; Yagita H.; Flavell R.; Boone Miller J.; Burkly L.; Zheng T. TWEAK, via its receptor Fn14, is a novel regulator of mesenchymal progenitor cells and skeletal muscle regeneration. EMBO J. 25: 5826-5839; 2006.

Jankowski R (2011) A comparison of commercially-available human skeletal muscle cells and media for research applications. Nature Methods/Application Notes. http://www.nature.com/app_notes/ nmeth/2011/111406/full/an7998.html.

Lecomte V.; Meugnier E.; Euthine V.; Durand C.; Freyssenet D.; Nemoz G.; Rome S.; Vidal H.; Lefai E. A new role for sterol regulatory element binding protein 1 transcription factors in the regulation of muscle mass and muscle cell differentiation. Mol. Cell. Biol. 30: 1182-1198; 2010.

Lee S. J. Regulation of muscle mass by myostatin. Annu. Rev. Cell Dev. Biol. 20: 61-86; 2004.

Lee S. J.; Reed L.; Davies M.; Girgenrath S.; Goad M.; Tomkinson K.; Wright J.; Barker C.; Ehrmantraut G.; Holmstrom J.; Trowell B.; Gertz B.; Jiang M.-S.; Sebald S.; Matzuk M.; Li E.; Liang L.; Quattlebaum E.; Stotish R.; Wolfman N. Regulation of muscle growth by multiple ligands signaling through activin type II receptors. Proc. Natl. Acad. Sci. U. S. A. 102: 18117-18122; 2005.

McFarlane C.; Plummer E.; Thomas M.; Hennebry A.; Murray A.; Ling N.; Smith H.; Sharma M.; Kambadur R. Myostatin induces cachexia by activating the ubiquitin proteolytic system through an
NF-kB-independent, FoxO1-dependent mechanism. J. Cell. Physiol. 209: 501-514; 2006.

Megeney L.; Rudnicki M. Determination versus differentiation and the MyoD family of transcription factors. Biochem. Cell Biol. 73: 723-732; 1995.

Ozernyuk N.; Balan O. Muscle satellite cells and regulation of recovery potential of muscle. Biol. Bull. 34: 542-550; 2007.

Pistilli E.; Bogdanovich S.; Goncalves M.; Ahima R.; Ahima R.; Lachey J.; Seehra J.; Khurana T. Targeting the activin type IIB receptor to improve muscle mass and function in the mdx mouse model of Duchenne muscular dystrophy. Am. J. Pathol. 178: 1287-1297; 2011.

Rebbapragada A.; Benchabane H.; Wrana J.; Celeste A.; Attisano L. Myostatin signals through a transforming growth factor beta-like signaling pathway to block adipogenesis. Mol. Cell. Biol. 23: 72307242; 2003.

Semsarian C.; Sutrave P.; Richmond D.; Graham R. Insulin-like growth factor (IGF-1) induces myotube hypertrophy associated with an increase in anaerobic glycolysis in a clonal skeletal-muscle cell model. Biochem. J. 339(Pt 2): 443-451; 1999.

Stitt T.; Drujan D.; Clarke B.; Panaro F.; Timofeyva Y.; Kline W.; Gonzalez M.; Yancopoulos G.; Glass D. The IGF-1/PI3K/Akt pathway prevents expression of muscle atrophy-induced ubiquitin ligases by inhibiting FOXO transcription factors. Mol. Cell 14: 395-403; 2004.

Sultan K.; Henkel B.; Terlou M.; Haagsman H. Quantification of hormone-induced atrophy of large myotubes from $\mathrm{C} 2 \mathrm{C} 12$ and L6 cells: atrophy-inducible and atrophy-resistant $\mathrm{C} 2 \mathrm{C} 12$ myotubes. Am. J. Physiol. Cell Physiol. 290: C650-C659; 2006.

Thies R.; Chen T.; Davies M.; Tomkinson K.; Pearson A.; Quazi S.; Wolfman N. GDF-8 propeptide binds to GDF-8 and antagonizes biological activity by inhibiting GDF-8 receptor binding. Growth Factors 18: 251-259; 2001.

Zammit P.; Partridge T.; Yablonka-Reuveni Z. The skeletal muscle satellite cell: the stem cell that came in from the cold. J. Histochem. Cytochem. 54: 1177-1191; 2006.

Zhao W.; Qin W.; Pan J.; Wu Y.; Bauman W.; Cardozo C. Dependence of dexamethasone-induced Akt/FOXO1 signaling, upregulation of MAFbx, and protein catabolism upon the glucocorticoid receptor. Biochem. Biophys. Res. Commun. 378: 668-672; 2009. 